\title{
Barcelona
}

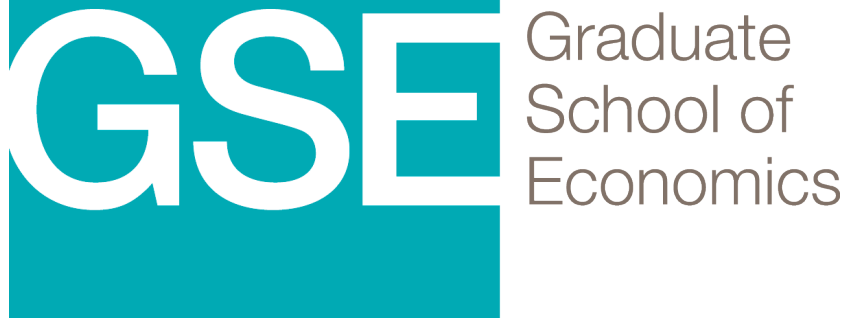

\section{Group Strategy-Proofness in Private Good Economies}

Salvador Barberà

Dolors Berga

Bernardo Moreno

This version: October 2015

(December 2014)

Barcelona GSE Working Paper Series

Working Paper $n^{\circ} 801$ 


\title{
Group strategy-proofness in private good economies
}

\author{
by \\ Salvador Barberà*, Dolors Berga ${ }^{\dagger}$, and Bernardo Moreno ${ }^{\ddagger}$
}

October, 2015

${ }^{*}$ Corresponding author. MOVE, Universitat Autònoma de Barcelona, and Barcelona GSE. Mailing address: Departament d'Economia i d'Història Econòmica, Edifici B, 08193 Bellaterra, Spain. E-mail: salvador.barbera@uab.cat

${ }^{\dagger}$ Departament d'Economia, C/ Universitat de Girona, 10; Universitat de Girona, 17071 Girona, Spain. E-mail: dolors.berga@udg.edu

${ }^{\ddagger}$ Departamento de Teoría e Historia Económica, Facultad de Ciencias Económicas y Empresariales, Campus de El Ejido, 29071 Málaga, Spain. E-mail: bernardo@uma.es 
Abstract: Many salient rules to allocate private goods are not only strategyproof, but also group strategy-proof, in appropriate domains of definition, hence diminishing the traditional conflict between incentives and efficiency. That is so for solutions to matching, division, cost sharing, house allocation and auctions, in spite of the substantive disparity between these cases. In a general framework encompassing all of them, we prove that the equivalence between the two forms of strategy-proofness is due to an underlying common structure, that transcends the many differences between the contexts and the mechanisms for which it holds.

Journal of Economic Literature Classification Numbers: C78, D71, D78.

Keywords: Matching, Division, House allocation, Cost sharing, Auctions, Strategyproofness, Richness, Group strategy-proofness, Joint monotonicity, Respectfulness, Nonbossiness. 


\section{Introduction}

In many contexts where satisfactory strategy-proof mechanisms do not exist, asking for more becomes redundant. But in domains where that basic incentive property can be met, it becomes natural to investigate whether there exist mechanisms that are not only immune to manipulation by individuals, but can also resist manipulation by groups of coordinated agents. ${ }^{1}$ This is particularly desirable for two reasons. One has to do with small coalitions. Individual strategy-proofness is a rather fragile property, unless one can also preclude manipulations of the social outcome by potential coalitions, especially if these may be sufficiently small and easy to coordinate. That danger is avoided under group strategy-proof mechanisms. The second reason is that, under group strategy-proofness, the grand coalition cannot get joint improvements, either. Therefore, this property guarantees efficiency $^{2}$, in addition to good incentives: these two desiderata, that are difficult to satisfy simultaneously in other contexts, become compatible.

In this paper we study the incentive properties of mechanisms to allocate private goods among selfish agents. We start from the striking observation that many well known individually strategy-proof mechanisms are also group strategy-proof, even if the latter is in principle a much stronger condition than the former. And this happens in situations that are formally modeled in rather different manners, including matching, division, cost sharing, house allocation and auctions. We want to determine whether this coincidence between the two a priori different incentive properties arises in each case for specific reasons, or whether there is a common ground for all of them.

In fact, strategy-proofness is a property of social choice functions, rather than a property of mechanisms. Agents that operate under a given mechanism may be using very general strategies, and the idea of truthtelling is not directly applicable to them. However, mechanisms where agents are endowed with dominant strategies are naturally associated with the direct mechanism where agent's messages are preferences: indeed, these are social choice functions, and they satisfy strategy-proofness. We make this remark for precision, though in what follows we shall abide with tradition and keep talking about strategy-proof mechanisms when this does not lead to confusion. Actually, the remark is relevant for another, more important reason. Thinking of dominant strategy mechanisms in terms of their direct

\footnotetext{
${ }^{1}$ We study that question for economies with public goods in Barberà, Berga, and Moreno (2010).

${ }^{2}$ As we shall see, several notions of group strategy-proofness will imply different levels of efficiency, but in all cases weak efficiency will at least be guaranteed.
} 
representation as social choice functions provides us with a common language to describe models that take very different forms, but share the same feature of being both individual and group strategy-proof in their respective setups. Some of these mechanisms operate in worlds where monetary transfers are excluded, like many-to-one matching (Gale and Shapley, 1962, see also Roth and Sotomayor, 1990), division problems (Sprumont, 1991) and house allocation in its simplest form (Shapley and Scarf, 1974). Others address problems where money transfers are possible: sharing the costs of public goods (Serizawa, 1999), matching with contracts (Hatfield and Kojima, 2009), house allocation with prices (Miyagawa, 2001) or auctions (Vickrey, 1961). Under some of these mechanisms only subsets of agents have the right incentives, while for others all of them do. For example, in matching models or in auctions, strategy-proofness in its different forms may be only satisfied for members of one side of the market, while in housing problems these properties apply to all participants. And there are many other differences among the models we are interested in. Yet, we identify properties that are sufficient to precipitate the group strategy-proofness of those social choice functions that are individually strategy-proof. All of our examples satisfy these properties, once specialized to fit the characteristics of each model. Hence, our equivalence result does not only clarify the intriguing connection between the two properties, but also proves that their link is independent of whether individual and group strategy-proofness hold for all agents or only for some.

The paper proceeds as follows. After this introduction, in Section 2 we present a general model, provide two allocation problems where attractive group strategy-proof mechanisms can be defined, and briefly discuss how each one of them fits the general framework. In Section 3 we identify three conditions that are satisfied by these two mechanisms and by many others: one is on the domain and the others are defined on the function itself. We then prove that for all mechanisms satisfying them, individual and group strategy-proofness become equivalent. In Section 4 we provide a larger set of applications where the allocation problem takes different forms and alternative mechanisms are proposed for each one, all of which are group strategy-proof. We briefly show how the applications fit our general framework and satisfy the conditions of our equivalence theorem. Finally, in Section 5 we place our contribution in further perspective, offer comments on related work by other authors, and discuss alternative questions of interest. The Appendix offers a collection of examples that illustrate several interesting relationships between conditions that we propose in the main text, and help to understand the role they play in our model and result. 


\section{The model and some leading applications}

Let $N=\{1,2, \ldots, n\}$ be a finite set of agents with $n \geqslant 2$. Let $B_{i}$ be the set of possible consequences for $i, i \in N$. Let $A \subseteq B_{1} \times \ldots \times B_{n}$ be the set of feasible combinations of consequences for agents and $a=\left(a_{1}, \ldots, a_{n}\right) \in A$. $A$ is our set of alternatives.

Each agent $i$ has preferences denoted by $R_{i}$ on $B_{i}$. As usual, we denote by $P_{i}$ and $I_{i}$ the strict and the indifference part of $R_{i}$, respectively. For any $a \in A$ and $R_{i}$, the strict lower contour set of $R_{i}$ at $a_{i}$ is $\bar{L}\left(R_{i}, a_{i}\right)=\left\{b_{i} \in B_{i}: a_{i} P_{i} b_{i}\right\}$ and the strict upper contour set of $R_{i}$ at $a_{i}$ is $\bar{U}\left(R_{i}, a_{i}\right)=\left\{b_{i} \in B_{i}: b_{i} P_{i} a_{i}\right\}$.

Let $\widetilde{\mathcal{R}}_{i}$ be the set of complete, reflexive, and transitive orderings on $B_{i}$. From preferences on $B_{i}$ we can induce preferences on $A$ as follows: For any $a, b \in A, a R_{i} b$ if and only if $a_{i} R_{i} b_{i}$. That is, we assume that, when evaluating different alternatives, agents are selfish. Note that, abusing notation we use the same symbol $R_{i}$ to denote preferences on $A$ and on $B_{i}$.

Let $\mathcal{R}_{i} \subseteq \widetilde{\mathcal{R}}_{i}$ be the set of admissible preferences for agent $i \in N$. A preference profile, denoted by $R_{N}=\left(R_{1}, \ldots, R_{n}\right)$, is an element of $\times_{i \in N} \mathcal{R}_{i}$. We will write $R_{N}=\left(R_{C}, R_{N \backslash C}\right) \in$ $\times_{i \in N} \mathcal{R}_{i}$ when we want to stress the role of coalition $C$ in $N$.

A social choice function (or a rule) is a function $f: \times_{i \in N} \mathcal{R}_{i} \rightarrow A$.

Let us first concentrate on incentive properties. The best known nonmanipulability axiom is that of strategy-proofness. In its usual form it requires the truth to be a dominant strategy for all agents. Our definition will be qualified, allowing for this requirement to only hold for agents in some group $H$.

Definition 1 A social choice function $f$ on $\times_{i \in N} \mathcal{R}_{i}$ is manipulable at $R_{N} \in \times_{i \in N} \mathcal{R}_{i}$ by agent $i \in N$ if there exists $R_{i}^{\prime} \in \mathcal{R}_{i}$ such that $R_{i}^{\prime} \neq R_{i}$ and $f\left(R_{i}^{\prime}, R_{N \backslash\{i\}}\right) P_{i} f\left(R_{N}\right)$. For $H \subseteq N$, a social choice function is $H$-strategy-proof if it is not manipulable by any agent $i \in H$.

A more demanding form of strategy-proofness is obtained by requiring that no group of individuals, of any size, could benefit from joint departures from truthful preference revelation. This is the general idea underlying the notion of group strategy-proofness. But while the formulation of the individual property is uncontroversial, here we face the possibility of defining several versions of the new property. In particular, we may specify in different manners what is meant by a group to benefit from strategic preference misrepresentation. This leads us to propose two alternative notions of group strategy-proofness. Under the first definition, we require that, in order to agree to manipulate, all participants 
must strictly gain from it. Since this is a strong requirement, the resulting notion of group strategy-proofness will be termed weak. In the second definition, we allow some members of a coalition to participate in a manipulation even if they do not make any gain from it (not a loss either), provided someone else in the group does. Since here it is easier to agree on a manipulation, we term the resulting notion of group strategy-proofness to be strong.

This is the spirit of the two definitions below. ${ }^{3}$

Definition $2 A$ social choice function $f$ on $\times_{i \in N} \mathcal{R}_{i}$ is strongly manipulable at $R_{N} \in$ $\times_{i \in N} \mathcal{R}_{i}$ by coalition $C \subseteq N$ if there exists $R_{C}^{\prime} \in \times_{i \in C} \mathcal{R}_{i}$ such that $f\left(R_{C}^{\prime}, R_{N \backslash C}\right) P_{i} f\left(R_{N}\right)$ for all $i \in C$. For $H \subseteq N$, a social choice function is $H$-weakly group strategy-proof if it is not strongly manipulable by any coalition $C \subseteq H$.

Definition 3 A social choice function $f$ on $\times_{i \in N} \mathcal{R}_{i}$ is weakly manipulable at $R_{N} \in \times_{i \in N} \mathcal{R}_{i}$ by coalition $C \subseteq N$ if there exists $R_{C}^{\prime} \in \times_{i \in C} \mathcal{R}_{i}$ such that $f\left(R_{C}^{\prime}, R_{-C}\right) R_{i} f\left(R_{N}\right)$ for all $i \in C$, and $f\left(R_{C}^{\prime}, R_{-C}\right) P_{j} f\left(R_{N}\right)$ for some $j \in C$. For $H \subseteq N$, a social choice function is $H$-strongly group strategy-proof if it is not weakly manipulable by any coalition $C \subseteq H$.

Notice that, in order to make sense, the definition of individual strategy-proofness requires that the domain should be a Cartesian product. Otherwise, the basic underlying idea that agents can decide what preferences to declare irrespectively of those declared by others could not be properly expressed. In the case of group manipulations, a bit more room is left, and the concept can be applied, in principle, to some non-Cartesian domains without doing violence to the idea of coordinated actions (see Penn, Patty, and Gailmard, 2011). Since our main purpose is to examine further properties that may be satisfied by rules that we assume to be strategy-proof from the onset, we restrict attention to the Cartesian domains where that basic condition is well defined.

It is worth insisting that all of our conditions are defined for any subset $H$ of agents, while their standard versions apply to the whole set of individuals involved in the allocation problem $(H=N)$. This is because, as we shall see, for some important problems, standard versions of those properties are essentially impossible to obtain, and yet some attractive and meaningful allocation methods satisfy them for at least some part of the agents. ${ }^{4}$ In

\footnotetext{
${ }^{3}$ Other definitions of group strategy-proofness and related notions are conceivable. See Section 5 for further discussion.

${ }^{4}$ This is the case in models where there are two differentiated types of agents, or sides of the market. For example, as we shall see, in classical matching models the Gale-Shapley mechanism only provides one side of
} 
these and in all other definitions where this qualification applies and $H$ is a strict subset of agents, we shall use the above expressions in full. When the properties apply to all agents, we may omit the reference to the set $H$.

Remark that, formally, strategy-proofness is a much weaker condition than group strategyproofness in any of its versions. Our challenge is to explain why in some cases this gap closes and both requirements become equivalent. This will, of course, depend on the notion of group strategy-proofness that we discuss. Here we'll concentrate on weak group strategyproofness because of the very nature of our exercise. We were surprised and interested by the fact that two apparently different incentive requirements can collapse into one. And this happens for many mechanisms. An important task is to understand the root of this coincidence, and we feel that the larger the class of mechanisms that we can put under the same umbrella, the more basic will the unifying conditions that we may discover to lie under all of them.

To prove the generality of our framework and the nature of our inquiry we present two problems and two paradigmatic mechanisms to solve them, out of the many cases covered by our main result. Each one of them is described in the literature in the usual language of its respective field. But to each mechanism we can associate a social choice function that directly links the preferences of participating agents with the outcome that would obtain from letting these agents operate within the mechanism if guided by such preferences. This associated social choice function is called the direct mechanism in the mechanisms design literature. It provides us with a natural language to examine the common traits of otherwise apparently diverse mechanisms in a unified manner. And it also allows us to emphasize how their many differences, in the form of a variety of domains, outcomes and strategies, are inconsequential for our main equivalence result. The applications we have selected to motivate our presentation are, as we shall see, two polar cases within the wide range of mechanisms that fall into our universe. Later on, in Section 4, we shall provide a similar analysis of other significant allocation problems and of some paradigmatic mechanisms to solve them.

One-to-one matching. (Gale and Shapley, 1962, Roth and Sotomayor, 1990) Agents are divided in two sides: workers and firms. Each firm can only hire one worker, and each worker can only work for one firm. Alternatives are matchings, assigning workers to

the market with dominant strategies to reveal the truth. By relaxing the standard definitions and allowing for partial notions of manipulation and of strategy-proofness, we can unify the treatments of matching problems and mechanisms with that of other cases that do no present the same asymmetries among agents. 
firms, in a reciprocal manner, and eventually leaving some agents matched with themselves (meaning that they remain unmatched to others). Possible consequences for an agent are the agents that she may be matched to. Preferences are initially defined over possible partners and oneself. They are typically assumed to be strict over these consequences. They naturally extend to alternatives (matchings) when agents are selfish. Notice that, then, many alternatives become indifferent to each other for an agent, as long as they assign her the same partner. The resulting admissible profiles are the domain of social choice functions selecting one matching for each combination of preferences. The celebrated Gale-Shapley algorithm selects a unique matching for each specification of preferences, as soon as one of the sides of the market (say the workers for the purpose of this illustration) is assigned the specific role of proposer. The algorithm works as follows. It starts with all workers applying to their preferred firms, and firms tentatively accepting the one worker they prefer among those that applied for it. If that leaves some workers unmatched, these are then asked to apply to their second best firms. Once their applications get in, firms may accept these new applicants if they are better for them than the ones they retained in the first round, and reject the previously accepted ones. That leads to a new matching and to a new list of unmatched workers. Again, if some workers remain unmatched, they may apply to that firm that is best among those they did not approached in preceding rounds. The process continues until no further changes occur. This always leads to a single alternative (matching).

The use of the Gale-Shapley mechanism at each preference profile generates a social choice function. We will abuse terminology and call it the Gale-Shapley social choice function from now on. Extensions of the basic model in different directions are possible, and they will also fall into the range of mechanisms our result applies to. ${ }^{5}$

Cost sharing of a public good (Serizawa, 1999, Moulin, 1994) As a second illustration, we consider a specific mechanism within a class that was characterized by Theorem 3 in Serizawa (1999), following lines initiated by Moulin (1994). A set of $n$ agents must decide on the amount of a public good to be produced, and how to share the costs of production of the agreed upon quantity. Consequences for each agents will be the amount of public good they will enjoy and the share of the cost that they will be charged. Given a cost function for production of the (unique) public good in terms of the (unique) private good, alternatives will be $n+1$ tuples indicating how much public good to be produced and how much private good must be contributed by each agent. Preferences of agents over

\footnotetext{
${ }^{5}$ These extensions are discussed in Section 4.
} 
alternatives are assumed to be increasing in both the private and the public good, continuous and convex. These preferences are naturally extended to alternatives when agents are selfish. Feasible alternatives consist of those where the contributions of agents cover exactly the cost of production of the public good. A mechanism assigns an alternative to every profile of preferences. Under the assumption that the cost function is concave, and that the agent knows the share of total cost it must pay, notice that the agent's preferences over consequences will be single-peaked (informally, each one will have a unique best amount of public good, and their utility will decrease with distance to this amount). Serizawa's simple scheme consists of announcing to all agents that they will have to equally share the cost of the public good, ask them to indicate their most desired amount, and then producing the minimum of the demanded quantities.

The mechanisms we just presented in these applications satisfy individual and group strategy-proofness with appropriate qualifications. Under the Gale-Shapley mechanism, declaring the true preferences is a dominant strategy for the proposers, hence, the associated direct mechanism (social choice function) is $P$-strategy proof, where $P$ stands for the set of agents on the side of the proposers. ${ }^{6}$ It is also true that no coalition formed by any subset of proposers can find a set of coordinated deviations from the truth that will guarantee that they all obtain a better outcome. The function is not only individual (see Dubins and Freedman, 1981 and Roth, 1982) but also weakly group strategy-proof (Dubins and Freedman, 1981). ${ }^{7}$ Notice, however, that the rule does not satisfy strong group strategyproofness. $^{8}$

Serizawa's cost sharing rule is individual and weak group strategy-proofness for the set of all agents (see Serizawa, 1999).

We have chosen these two simple applications to start with, because they are quite different even if our equivalence holds for both. In the matching case, only some agents have dominant strategies, while in the cost sharing application all agents do. In one case group strategy-proofness is weak, while strong in the other. The domain of preferences is restricted in both cases by the selfishness assumption, but otherwise different. In the case of matching preferences over consequences are unrestricted. In the cost sharing application,

\footnotetext{
${ }^{6}$ However, the agents in the other side of the market can manipulate by misrepresenting their preferences.

${ }^{7}$ The original proofs of these facts where nontrivial. The equivalence between individual and group strategy-proofness for specific mechanisms continues to be the object of recent papers. See for example Martínez, Massó, Neme, and Oviedo (2004) and Hatfield and Kojima (2009).

${ }^{8}$ For an example proving this fact and further discussion, see Example 7 in the Appendix and Section 5 , respectively.
} 
the combination of preference convexity and concave costs implies single-peakedness over the amount of public good. Finally, remark that in the case of matching, the best consequence for an agent is well defined, whereas in the cost sharing case for any given allocation there is always a better one.

In the next section we shall dig into the foundations that make these and many other different allocation problems to share the same equivalence. To do that we adopt a general enough view that takes into account the differences between mechanisms that we have hinted at, but concentrate on the essential similarities of their associated direct mechanisms.

\section{The equivalence between individual and weak group strategy-proofness}

We now present the three conditions that will be used to establish the equivalence between individual and weak group strategy-proofness. We have identified these conditions after close examination of a wide variety of mechanisms that share the equivalence, since they appear as a natural common denominator for all of them. And we shall argue that they are, in addition, conditions of independent normative interest. Let us now express them formally.

First, we ask for the domain to satisfy a richness condition.

Definition 4 A set of individual preferences $\mathcal{R}_{i}$ is rich if for any $R_{i}, \widetilde{R}_{i} \in \mathcal{R}_{i}, a_{i}, b_{i} \in B_{i}$ such that $b_{i} P_{i} a_{i}$,

there exists $R_{i}^{\prime} \in \mathcal{R}_{i}$ such that $\bar{U}\left(R_{i}^{\prime}, b_{i}\right) \subseteq \bar{U}\left(R_{i}, b_{i}\right) \cap \bar{U}\left(\widetilde{R}_{i}, b_{i}\right), \bar{L}\left(\widetilde{R}_{i}, b_{i}\right) \subseteq \bar{L}\left(R_{i}^{\prime}, b_{i}\right)$, $\bar{U}\left(R_{i}, a_{i}\right)=\bar{U}\left(R_{i}^{\prime}, a_{i}\right)$ and $\bar{L}\left(R_{i}, a_{i}\right)=\bar{L}\left(R_{i}^{\prime}, a_{i}\right)$.

Definition 5 Let $H \subseteq N$. A domain of preferences $\times_{i \in N} \mathcal{R}_{i}$ is $H$-rich if for any $i \in H$, $\mathcal{R}_{i}$ is rich.

Remark that, in our case, the idea of a rich domain is based on a certain notion of betweenness: if two preferences are admissible for an agent, then a third preference that combines their characteristics in a specific manner must also be admissible. As we shall see, many natural domains meet the condition. However there are other domains that do 
not, like those of separable preferences. But then it is known that mechanisms operating in such domains are typically inefficient and not weak group strategy-proof. ${ }^{9}$

The condition has an easy implication when the preferences are such that each agent has always a best consequence, and all possible consequences can be best. This is the case, for example, when there is a finite set of objects and preferences are linear orders of objects, like in matching, or even in some cases where the set of consequences is infinite, like in the division problem under single-peaked preferences over shares. Then, given any $R_{i}, \widetilde{R}_{i}, a_{i}, b_{i}$ we can check whether there exists an $R_{i}^{\prime}$ where $a_{i}$ stays at the same position than in $R_{i}$ but $b_{i}$ ranks at the top (see this simpler condition in Barberà, Berga, and Moreno, 2014). The reason why we do not postulate our domain restrictions in these simpler terms is that, in our present formulation, we can also include problems where the notion of a best element is not well defined, but our condition holds and is even easy to check for. This is the case, for example, in allocation problems where we admit unbounded monetary compensations.

Next, we ask our functions to respond positively to preference changes, in a natural and rather weak form. This is reflected by the notion of $H$-joint monotonicity. It requires the following. Given a set $H$ of agents, and a preference profile $R_{N}$, consider the profile $R_{N}^{\prime}$ where $f_{i}\left(R_{N}\right)$ has improved its position for some agents $i$ in $H$. Then, $f\left(R_{N}^{\prime}\right)$ should have a consequence that is as good as the one obtained by these agents at $f\left(R_{N}\right)$.

Formally,

Definition 6 Let $H \subseteq N$ be a subset of agents. A social choice function $f$ satisfies $H$-joint monotonicity on $\times_{i \in N} \mathcal{R}_{i}$ if for any $R_{N} \in \times_{i \in N} \mathcal{R}_{i}, C \subseteq H$, and $R_{C}^{\prime} \in \times_{i \in C} \mathcal{R}_{i}\left(R_{i}^{\prime} \neq R_{i}\right.$ for any $i \in C)$ such that $\bar{L}\left(R_{i}, f_{i}\left(R_{N}\right)\right) \subseteq \bar{L}\left(R_{i}^{\prime}, f_{i}\left(R_{N}\right)\right)$ and $\bar{U}\left(R_{i}, f_{i}\left(R_{N}\right)\right) \supseteq \bar{U}\left(R_{i}^{\prime}, f_{i}\left(R_{N}\right)\right)$ for each $i \in C$, then $f_{i}\left(R_{C}^{\prime}, R_{N \backslash C}\right) R_{i}^{\prime} f_{i}\left(R_{N}\right)$ for each $i \in C$.

This condition belongs to a class of requirements that admit several formulations but share the same motivation: if one agent gets a consequence and then that consequence becomes more appreciated by her than it was, this change cannot work against the agent's interest.

A relatively strong requirement within this class is known as Maskin's monotonicity, and ours is strictly weaker than this well known condition. ${ }^{10}$ At the other end, some notions of monotonicity are only required to apply when the improvement of the initially chosen

\footnotetext{
${ }^{9}$ For example, the Vickrey-Clarke-Groves (VCG) mechanism, or the voting by committees procedures defined in Barberà, Sonneschein, and Zhou (1991).

${ }^{10}$ For a complete argument and more comments about this fact, see Section 5
} 
alternative is in fact the only change between the two profiles that are compared: ours is stronger than those, since it applies even when the ranking of consequences within the strict upper and lower contour sets can vary.

Our last condition establishes some limits on the impact that changes in the preferences of one agent can have on the consequences that the mechanism assigns to others. We call it $H$-respectfulness. It requires that, whenever an agent changes preferences in a limited manner, and this has no consequences on her utility, then the utilities of other agents remain unchanged.

Specifically,

Definition 7 Let $H \subseteq N$ be a subset of agents. A social choice function $f$ on $\times_{i \in N} \mathcal{R}_{i}$ is $H$-respectful if

$$
f_{i}\left(R_{N}\right) I_{i} f_{i}\left(R_{i}^{\prime}, R_{N \backslash\{i\}}\right) \text { implies } f_{j}\left(R_{N}\right) I_{j} f_{j}\left(R_{i}^{\prime}, R_{N \backslash\{i\}}\right), \forall j \in H \backslash\{i\},
$$

for each $i \in H, R_{N} \in \times_{i \in N} \mathcal{R}_{i}$, and $R_{i}^{\prime} \in \mathcal{R}_{i}$ such that $\bar{U}\left(R_{i}, f_{i}\left(R_{N}\right)\right)=\bar{U}\left(R_{i}^{\prime}, f_{i}\left(R_{N}\right)\right)$ and $\bar{L}\left(R_{i}, f_{i}\left(R_{N}\right)\right)=\bar{L}\left(R_{i}^{\prime}, f_{i}\left(R_{N}\right)\right){ }^{11}$

Like the preceding one this condition has normative appeal. Under appropriate conditions, no agent should be able to change the utility of others without changing her own. For short, we say that the type of changes in individual preferences contemplated in Definition 7 are reshufflings of the initial preferences around a given consequence.

The condition of $H$-respectfulness is in a similar spirit, but much weaker than others also requiring that agents's preference changes that have no impact on them should not have consequences on others. A seminal definition in this vein was that of non-bossiness (Satterthwaite and Sonneschein, 1981).

Definition 8 A social choice function $f$ on $\times_{i \in N} \mathcal{R}_{i}$ is non-bossy if

$$
f_{i}\left(R_{i}, R_{N \backslash\{i\}}\right)=f_{i}\left(\widetilde{R}_{i}, R_{N \backslash\{i\}}\right) \text { implies } f\left(R_{i}, R_{N \backslash\{i\}}\right)=f\left(\widetilde{R}_{i}, R_{N \backslash\{i\}}\right)
$$

for any $i \in N$, any $R_{i}, \widetilde{R}_{i} \in \mathcal{R}_{i}$, and any $R_{N \backslash\{i\}} \in \times_{i \in N \backslash\{i\}} \mathcal{R}_{i}$.

\footnotetext{
${ }^{11}$ Notice that our requirement is restricted to changes in the mechanisms outcomes that are induced by a limited class of preference changes. Under strategy-proof mechanisms these changes will have no utility impact on the individual whose preferences are modified. This is because the reshufflings above and below the indifference sets of the initial outcome cannot improve or worsen the utility of an agent under strategy-proofness.
} 
Notice that when preferences over consequences are strict, non-bossiness implies respectfulness. Even in some frameworks where indifferences are allowed, the same implication may also hold in conjunction with additional properties (see the case of the division problem in Section 4).

Another proposal that implies respectfulness was made by Ritz (1985), in a form that requires consequences for others not to change, if one's utilities are not changed.

Definition 9 A social choice function $f$ on $\times_{i \in N} \mathcal{R}_{i}$ is noncorruptible if

$f_{i}\left(R_{i}, R_{N \backslash\{i\}}\right) I_{i} f_{i}\left(\widetilde{R}_{i}, R_{N \backslash\{i\}}\right)$ and $f_{i}\left(R_{i}, R_{N \backslash\{i\}}\right) \widetilde{I}_{i} f_{i}\left(\widetilde{R}_{i}, R_{N \backslash\{i\}}\right)$ implies $f\left(R_{i}, R_{N \backslash\{i\}}\right)=f\left(\widetilde{R}_{i}, R_{N \backslash\{i\}}\right)$

for any $i \in N$, any $R_{i}, \widetilde{R}_{i} \in \mathcal{R}_{i}$, and any $R_{N \backslash\{i\}} \in \times_{i \in N \backslash\{i\}} \mathcal{R}_{i}$.

And there are other similar conditions, which have been recently surveyed by Thomson (2014).

Now, before stating and proving our main result, let us check that the three conditions we propose as a common denominator among many mechanisms will be satisfied by those two we have already presented in the previous section.

Let's start by the Gale-Shapley mechanism. It is trivial to see that our domain condition is satisfied in the standard version of the matching problem, since all possible orderings among consequences are admissible for each agent. To argue that $H$-joint monotonicity and $H$-respectfulness are satisfied, with $H$ being the set of proposers, we first refer to two important properties of the mechanism. One is that it always selects a stable outcome. ${ }^{12}$ The other is that this outcome assigns to all agents in the proposers' side the best possible match among those that she can get at any stable matching. Indeed, the set of stable matchings is a lattice, and the stable alternative selected by the mechanism is the proposer's best. $^{13}$ Now, to show that the mechanism satisfies $H$-joint monotonicity, start from its image at any preference profile, and compare it with the resulting choice when a group of agents on the proposers' side, $H$ (which is either $W$ or $F$ ) have increased the valuation they attribute to the matches they were getting. Notice that the old matching will still be stable under the new preferences. This new matching is the best for the new proposers among all stable ones. But then, it will also be the best for the proposers under the initial preferences:

\footnotetext{
${ }^{12} \mathrm{~A}$ matching is stable if it is not blocked by any individual or any pair of agents. A matching is blocked by an individual if she prefers to be single rather than with her match. And a matching is blocked by a pair of agents if they both prefer to be together than with their respective matches.

${ }^{13}$ See Roth and Sotomayor (1990), Theorem 2.12 and Theorem 2.16.
} 
hence the two consequences will be the same for agents in $H$, and $H$-joint monotonicity holds.

A similar argument works to prove $H$-respectfulness. ${ }^{14}$ Now, if agents' preferences have undergone a reshuffling of the alternatives that were strictly above or below the indifference class to which the matching belongs, it will remain stable. But then, it will be the proposers' best stable matching for both the initial and the new preferences, and it will be chosen at both profiles. The individual whose preferences have changed will see no change in consequences, nor will anyone else.

We now turn to the second application: Serizawa's cost sharing method, in the context of concave cost functions and continuous, strictly convex preferences that are increasing in the amounts of public and private good. Now the notion of an absolute best alternative is not well defined. To prove richness, consider any pair of preferences $R_{i}, \widetilde{R}_{i} \in \mathcal{R}_{i}$, and any pair of consequences $a_{i}, b_{i} \in B_{i}$ such that $b_{i} P_{i} a_{i}$. Let $R_{i}^{\prime} \in \mathcal{R}_{i}$ be such that its indifference curve through $a_{i}$ is the same as that of $R_{i}$, and such that the upper contour set at $b_{i}$ is the intersection of the upper contour sets of $R_{i}$ and $\widetilde{R}_{i}$ at $b_{i}$. This preference is clearly admissible and satisfies the requirement imposed by richness.

To argue that joint monotonicity and respectfulness are satisfied, we use the following observation. For any given cost function and knowing that cost sharing will be egalitarian, each agent has an optimal combination of public good provision and share, which in fact can be expressed as a single-peaked preference over the amount of public good. Conversely, given any amount of public good, we can find preferences over the amount of it and the cost payments that would generate a preference that peaks at that given amount.

Joint monotonicity is clearly satisfied. An agent demanding the smallest amount of the public good determines the allocation at each profile. Any group of agents not containing this minimum demander, and whose members' strict upper contour set at the initial outcome decreases, will still demand a higher amount of public good and thus not change the initial outcome. Yet, the low demand agent will not change her choice of public good level when her upper contour shrinks. Therefore, the allocation of production and cost shares will be invariant to the predicated preference changes.

For very similar reasons, respectfulness will also hold. Any agent different from the minimum demander and whose strict upper and lower contour set at the initial outcome remain unchanged, will still demand a higher amount of public good with respect to that of

\footnotetext{
${ }^{14}$ However, the Gale-Shapley social choice function is bossy because that condition does not require changes of preferences to be reshufflings. See Example 7 in the Appendix.
} 
the low demand agent and thus not change the initial outcome. Yet, the low demand agent will not change her choice of public good level when her strict lower and upper contour set changes. Therefore, the allocation of production and cost shares will be invariant to the predicated preference changes of any agent.

We hope that these remarks on our first two applications (more will follow in Section 4), not only help the reader to test the proposed conditions against these particular cases, but also as an implicit argument that, in many instances, checking for these properties is a simple matter, probably more than directly checking for its performance regarding weak group strategy-proofness.

Let us now turn to our main theorem. It proves that the equivalence between that property and individual strategy-proofness is not a lucky coincidence, but a result of the fact that any strategy-proof social choice function satisfying the three common requisites that we just exhibited for individuals in a set $H$ must also be immune to strong manipulation by subsets of $H$.

Theorem 1 If $f$ is $H$-joint monotonic, $H$-respectful and defined on an $H$-rich domain, then it is $H$-strategy-proof if and only if it is $H$-weak group strategy-proof.

Proof Obviously, $H$-weak group strategy-proofness implies $H$-strategy-proofness. To prove the converse, suppose by contradiction that there exists $R_{N} \in \times_{i \in N} \mathcal{R}_{i}, C \subseteq H$, $\widetilde{R}_{C} \in \times_{i \in C} \mathcal{R}_{i}$ such that for any agent $i \in C, f_{i}\left(\widetilde{R}_{C}, R_{N \backslash C}\right) P_{i} f_{i}\left(R_{N}\right)$. Let $b=f\left(\widetilde{R}_{C}, R_{N \backslash C}\right)$ and $a=f\left(R_{N}\right)$. Without loss of generality, let $C=\{1, \ldots, c\}$.

First, we recursively define the preferences $R_{i}^{\prime}$ for $i$ in $C$. For $i=1$, let $R_{1}^{\prime}$ be such that $\bar{U}\left(R_{1}^{\prime}, b_{1}\right) \subseteq \bar{U}\left(R_{1}, b_{1}\right) \cap \bar{U}\left(\widetilde{R}_{1}, b_{1}\right), \bar{L}\left(R_{1}, a_{1}\right)=\bar{L}\left(R_{1}^{\prime}, a_{1}\right)$, and $\bar{U}\left(R_{1}, a_{1}\right)=\bar{U}\left(R_{1}^{\prime}, a_{1}\right)$. By $H-$ richness, such a preference $R_{1}^{\prime}$ is in $\mathcal{R}_{1}$. For $k \in C \backslash\{1\}$, define $a_{k}^{\prime}=f_{k}\left(R_{\{1, \ldots, k-1\}}^{\prime}, R_{N \backslash\{1, \ldots, k-1\}}\right)$ and $R_{k}^{\prime}$ to be such that $\bar{U}\left(R_{k}^{\prime}, b_{k}\right) \subseteq \bar{U}\left(R_{k}, b_{k}\right) \cap \bar{U}\left(\widetilde{R}_{k}, b_{k}\right), \bar{L}\left(R_{k}, a_{k}^{\prime}\right)=\bar{L}\left(R_{k}^{\prime}, a_{k}^{\prime}\right)$, and $\bar{U}\left(R_{k}, a_{k}^{\prime}\right)=\bar{U}\left(R_{k}^{\prime}, a_{k}^{\prime}\right)$. By $H$-richness, such a preference $R_{k}^{\prime}$ is in $\mathcal{R}_{k}$.

Let us now change one by one the preferences of each agent in $C$ from $R_{i}$ to $R_{i}^{\prime}$. By strategy-proofness applied to agent $1: f_{1}\left(R_{1}^{\prime}, R_{N \backslash\{1\}}\right) I_{1} f_{1}\left(R_{N}\right)=a_{1}$, otherwise agent 1 would manipulate $f$ at $\left(R_{N}\right)$ via $R_{1}^{\prime}$ or at $\left(R_{1}^{\prime}, R_{N \backslash\{1\}}\right)$ via $R_{1}$. By $H$-respectfulness, $f_{j}\left(R_{1}^{\prime}, R_{N \backslash\{1\}}\right) I_{j} f_{j}\left(R_{N}\right)=a_{j}$ for each agent $j \in H \backslash\{1\}$. Hence, for $j \in H \backslash\{1\}$, by transitivity of $R_{j}, b_{j} P_{j} f_{j}\left(R_{1}^{\prime}, R_{N \backslash\{1\}}\right)$. And by transitivity of $R_{1}^{\prime}$, since $\bar{L}\left(R_{1}, a_{1}\right)=\bar{L}\left(R_{1}^{\prime}, a_{1}\right)$ and $\bar{U}\left(R_{1}, a_{1}\right)=\bar{U}\left(R_{1}^{\prime}, a_{1}\right)$ then $b_{1} P_{1}^{\prime} f_{1}\left(R_{1}^{\prime}, R_{N \backslash\{1\}}\right)$.

A similar argument applies when the profile changes from $\left(R_{\{1, \ldots, k-1\}}^{\prime}, R_{N \backslash\{1, \ldots, k-1\}}\right)$ to $\left(R_{\{1, \ldots, k\}}^{\prime}, R_{N \backslash\{1, \ldots, k\}}\right)$, for $k=2, \ldots, c$. Again, by strategy-proofness applied to agent $k$, 
$f_{k}\left(R_{\{1, \ldots, k\}}^{\prime}, R_{N \backslash\{1, \ldots, k\}}\right) I_{k} f_{k}\left(R_{\{1, \ldots, k-1\}}^{\prime}, R_{N \backslash\{1, \ldots, k-1\}}\right)=a_{k}^{\prime}$.

By $H$-respectfulness, $f_{j}\left(R_{\{1, \ldots, k\}}^{\prime}, R_{N \backslash\{1, \ldots, k\}}\right) I_{j} f_{j}\left(R_{\{1, \ldots, k-1\}}^{\prime}, R_{N \backslash\{1, \ldots, k-1\}}\right)$ for each agent $j \in$ $H \backslash\{1, \ldots, k\}$ and $f_{l}\left(R_{\{1, \ldots, k\}}^{\prime}, R_{N \backslash\{1, \ldots, k\}}\right) I_{l}^{\prime} f_{l}\left(R_{\{1, \ldots, k-1\}}^{\prime}, R_{N \backslash\{1, \ldots, k-1\}}\right)$ for each agent $l \in\{1, \ldots, k-$ $1\}$. Therefore, by transitivity of $R_{j}, b_{j} P_{j} f_{j}\left(R_{\{1, \ldots, k\}}^{\prime}, R_{N \backslash\{1, \ldots, k\}}\right)$ for each agent $j \in H \backslash\{1, \ldots, k\}$. By transitivity of $R_{l}^{\prime}, b_{l} P_{l}^{\prime} f_{l}\left(R_{\{1, \ldots, k\}}^{\prime}, R_{N \backslash\{1, \ldots, k\}}\right)$ for each agent $l \in\{1, \ldots, k-1\}$. And by transitivity of $R_{k}^{\prime}$, since $\bar{L}\left(R_{k}, a_{k}^{\prime}\right)=\bar{L}\left(R_{k}^{\prime}, a_{k}^{\prime}\right), \bar{U}\left(R_{k}, a_{k}^{\prime}\right)=\bar{U}\left(R_{k}^{\prime}, a_{k}^{\prime}\right)$ then $b_{k} P_{k}^{\prime} f_{k}\left(R_{\{1, \ldots, k\}}^{\prime}, R_{N \backslash\{1, \ldots, k\}}\right)$.

Repeating the same argument for each agent in $C$ we obtain that

(1) $b_{j} P_{j}^{\prime} f_{j}\left(R_{C}^{\prime}, R_{N \backslash C}\right)$ for each agent $j \in C$.

We could have also arrived at the profile $\left(R_{C}^{\prime}, R_{N \backslash C}\right)$ from $\left(\widetilde{R}_{C}, R_{N \backslash C}\right)$. Notice that by construction of the $R_{i}^{\prime}$ we have that for each $i \in C, \bar{L}\left(\widetilde{R}_{i}, f_{i}\left(\widetilde{R}_{N}\right)\right) \subseteq \bar{L}\left(R_{i}^{\prime}, f_{i}\left(\widetilde{R}_{N}\right)\right)$ and $\bar{U}\left(\widetilde{R}_{i}, f_{i}\left(\widetilde{R}_{N}\right)\right) \supseteq \bar{U}\left(R_{i}^{\prime}, f_{i}\left(\widetilde{R}_{N}\right)\right)$. Thus, we can apply $H$-joint monotonicity and obtain that

(2) $f_{i}\left(R_{C}^{\prime}, R_{N \backslash C}\right) R_{i}^{\prime} f_{i}\left(\widetilde{R}_{C}, R_{N \backslash C}\right)=b_{i}$ for each $i \in C$.

By (1) and (2) we get the desired contradiction.

Our main purpose in this paper is achieved: we have identified sufficient conditions to guarantee the equivalence between our two incentive properties. ${ }^{15}$ We have already seen that these conditions hold in our two applications and later on we will exhibit other environments and mechanisms where the theorem also applies.

\section{Some applications}

In this section we discuss how the different allocation problems that we mentioned in the introduction actually fit the general framework we just defined. Since we are just trying to illustrate the fact that the model encompasses many special cases, we do not seek full generality. Rather, we try to describe the specific formulation of these problems as they appear in representative papers within each of the fields we mean to cover, and refer to these papers at each point.

Matching (Gale and Shapley, 1962, Roth and Sotomayor, 1990, Kelso and Crawford, 1982, Hatfield and Milgrom, 2005) We have already presented the basic Gale-Shapley mechanism to solve one-to-one matching problems in Sections 2 and 3. Here we discuss how it

\footnotetext{
${ }^{15}$ See Examples 1 to 3 in the Appendix where we show how violations of our assumptions may lead to failure of the equivalence. In addition Example 4 shows that $H$-respectfulness and $H$-joint monotonicity do not imply strategy-proofness; while Examples 5 and 6 prove that $H$-weak group strategy-proofness does not imply $H$-respectfulness, nor $H$-joint monotonicity.
} 
can be extended to cover more general cases, and prove that our theorem also applies to the enlarged set of environments and to the adapted new mechanisms. A traditional extension is to the case of many-to-one matchings. Now each worker can still only be matched to one firm, but firms may hire more than one worker, up to a personalized quota. A further and more recent extension has considered the introduction of contracts between workers and firms. There, the set of agents is enlarged. For a firm, the same worker under a different contract is a different agent. Similarly, the same firm offering a different contract is also treated as a different agent by the workers. Again, an alternative is a matching, which now specifies which physical agent goes with whom, as well as the contract that holds between the firms and workers that are matched. Consequences for workers are the firm she is matched to and the contract she works under. Consequences for firms are the workers they get, and their respective contracts. Workers have preferences over firm-contract pairs, and firms have preferences over worker-contract pairs. It is assumed that the rankings of these pairs are strict for agents on both sides of the market. The preferences of firms over sets of worker-contracts within their quota are assumed to satisfy specific conditions, connecting the preferences over individual pairs with those of sets of such pairs, in order to guarantee that the outcome of the extended Gale-Shapley method we'll describe in a moment will be stable. Here we assume that the firm's preferences over sets of workers satisfy the following condition of responsiveness: Assume that worker $s$ is preferred to worker $t$, when comparing them as singletons. Then, for any two sets sharing the same workers, except that one contains $s$ and the other contains $t$, the former is preferred to the latter. We concentrate on that domain of preferences because, as we shall see, it guarantees that the Gale-Shapley algorithm, originally designed for one-to-one matchings, can be used in that more general case and provide us with a rule satisfying the good properties we are after. ${ }^{16}$ These preferences are naturally extended to alternatives under the selfishness assumption, and their set constitutes the domain of the relevant social choice problems. Now, the way to extend the Gale-Shapley mechanism when workers propose is to consider that whenever they are free, they propose to the firm that, combined with a given contract, is best among those that had not yet rejected them. As for firms, they can be subdivided into identical units, each of which can only accept one worker with a given contract. That essentially reduces the problem to that of finding a one-to-one matching, by using the original Gale-Shapley

\footnotetext{
${ }^{16}$ Other conditions like substitutability and $q$-separability or the law of demand have also been used, alone or in combination, to extend the preferences from single consequences to sets of them. See Martínez, Massó, Neme, and Oviedo (2004) or Hatfield and Milgrom (2005).
} 
mechanism. It is clear that the added complexity of the set of consequences, and of the admissible preferences over them still allows for the domain to be $H$-rich, $H$ being the set of workers. As for the properties of $H$-joint monotonicity and $H$-respectfulness, the same arguments that we used in our discussion of the one-to-one matching mechanism still hold, because the essential fact that the outcome is the worker's best among all stable matchings continues to be true.

Cost sharing of a public good (Serizawa, 1999) The application we already discussed in the preceding sections is part of a larger class, all of whose members also satisfy the conditions of our theorem. Again, a set of individuals must select an amount of public good and share its production cost. Equal cost share is still retained, and can be derived from basic assumptions on the allocation rule. Thus, allocations, consequences, alternatives, and preferences remain the same as in the case we already discussed in Sections 2 and 3. However, given the agents' expressed demands, we can define many mechanisms that differ in the way in which the amount of public good is selected. Since the preferences of agents over possible levels of public good provision are single-peaked, the class of strategy-proof rules to determine the chosen amount is the one characterized by Moulin (1980). They include the minimal demand rule we already used in the simple application of Sections 2 and 3 , but also the maximal and the median rule, among many others. All these rules are also weakly group strategy-proof, and so are the mechanisms that result from using any one of these methods and then distributing the costs equally.

To show that these mechanisms also meet our conditions, notice that the argument for richness is identical than in the simple case. To argue that they all must satisfy joint monotonicity, notice that any shrinking of the upper contour sets above an initial consequence will leave the optimal demand of the public good of that agent on the same side of the line than it was, relative to the initially chosen level of that good. But then, these changes never affect the outcome of any of the strategy-proof mechanisms described by Moulin. ${ }^{17}$ Finally the mechanisms are non-bossy, hence respectful, since clearly no agent can stay indifferent and change the outcome for others.

Division under single-peaked preferences (Sprumont, 1991) A set of individuals must share a task. An alternative is an allocation, which is a vector of shares, indicating what proportion of the total task is assigned to each individual. Given an allocation, the

\footnotetext{
${ }^{17}$ Functions satisfying this condition are called uncompromising, a term coined by Border and Jordan (1983).
} 
consequence for an agent is just the share of the task she is assigned. Each agent is assumed to have single-peaked preferences on consequences, which can be extended to allocations. These extended preferences are the domain of social choice rules for the division problem.

The best known method to solve the division problem is the uniform rule. Here is how it works. Ask agents for their preferred share of the job. If the sum of the desired shares exceeds one, find a number $\lambda$ with the following property. If all agents who demand less than $\lambda$ are allowed to have their preferred share, and all others are required to accept $\lambda$, then the total assignment adds up to one. If the sum of the desired shares is short of one, find a number $\lambda^{\prime}$ having the following property. If all agents who demand more than $\lambda^{\prime}$ are allowed to have their preferred share, and all others are required to accept $\lambda^{\prime}$, then the total assignment adds up to one. These values for $\lambda$ or $\lambda^{\prime}$ always exist, and thus the rule based on them determines an assignment of shares that is always feasible. It therefore defines a social choice function on the set of admissible profiles. Given a profile of single-peaked preferences over shares of the job, the rule determines a unique alternative, that is, a vector of shares. Our social choice function is induced by assigning this unique proposal to each preference profile.

The uniform rule satisfies individual and group strategy-proofness (see Sprumont, 1991 and Barberà, Jackson, and Neme, 1997).

The family of all single-peaked preferences is rich because given any $a_{i}, b_{i} \in B_{i}$ such that $b_{i} P_{i} a_{i}$, one can trivially find another single-peaked preference $R_{i}^{\prime} \in \mathcal{R}_{i}$ with $b_{i}$ being the best consequence of $R_{i}^{\prime}$ and such that $\bar{U}\left(R_{i}, a_{i}\right)=\bar{U}\left(R_{i}^{\prime}, a_{i}\right), \bar{L}\left(R_{i}, a_{i}\right)=\bar{L}\left(R_{i}^{\prime}, a_{i}\right)$.

To show respectfulness and joint monotonicity, observe that the uniform rule is nonbossy and efficient. By efficiency, the changes in agents' preferences required in the hypothesis of respectfulness are such that an agent could be indifferent if she gets the same consequence. This fact together with non-bossiness implies respectfulness. To check for joint monotonicity, assume that only one agent changes her preferences that her initial consequence become better. By efficiency, the new and the old consequences are on the same side of her best consequence. Strategy-proofness imposes that the old and new consequences must be the same for that agent. By non-bossiness the consequence for other agents don't change either. Thus, non-bossiness allows us to treat joint changes by several agents as a chain of individual changes, none of which modifies the chosen alternative. Hence, joint monotonicity is satisfied.

House allocation (Shapley and Scarf, 1974, Miyagawa, 2001) A set of individuals must 
be assigned a maximum of one house each, out of a set of houses, and an eventual payment in the form of a divisible private good (money). ${ }^{18}$ An allocation fully specifies who gets what house, if any, and a payment for each individual. The consequence of an allocation for each agent are given by the house she gets and how much she pays.

Individuals have preferences over houses and money. For the same amount of payment, they are typically assumed to prefer any house to none, and their preferences over houses are otherwise unrestricted. Their preferences are assumed to be increasing in money. Houses are not agents, as they are not endowed with preferences. So now individuals are the agents, alternatives are allocations of at most one house to each agent, plus their individual payments, and social choice functions are defined over the domain of all quasi-linear preferences with respect to money, and unrestricted rankings of houses.

The case where no monetary transfers are allowed was the one to be studied first. Then allocations just specify who gets what house, consequences for agents are simply the house they get, and preferences over houses are typically unrestricted. All linear orders of the houses by agents are allowed, and their natural extension to allocations under the selfishness assumption constitute the domain of definition of the social choice function.

For the housing problem without money transfers (Shapley and Scarf, 1974), the top trading cycle mechanism also determines a unique alternative, this time an allocation of houses based on the strict preferences of agents over them. Here is how the method works. Ask agents to point at their preferred house. There will always be some set of agents (maybe several) whose demands form a cycle. Agents who point at their own house, form a cycle by themselves. Give those agents in the cycle their preferred houses and remove them. Now ask the remaining set of agents for their preferred houses over the remaining ones, and proceed likewise until all houses are assigned. The final allocation generates what we call, abusing terminology, the top trading cycle social choice function, which is well known to be strategy-proof and weak group strategy-proof (see Roth, 1982, Bird, 1984).

In that version of the housing problem, no restrictions are imposed on the agents' preferences over houses, thus the richness condition is satisfied.

To show respectfulness and joint monotonicity, observe that the top trading cycle is non-bossy (see Pápai, 2000). Since indifferences are not allowed, non-bossiness implies

\footnotetext{
${ }^{18}$ Since we are using these models for motivational purposes, we stick to the simplest version of the house allocation model. More complex cases allow for more than one house to be allocated to the same agents, the existence of property rights and other possible variations (see for example, Pápai, 2000, Ergin, 2002, and Velez, 2014).
} 
respectfulness. In the case of joint monotonicity, it is clearly satisfied by the top trading cycle, since any agent endowed with some house at a certain profile will retain that house if it eventually becomes higher in her ranking. Notice that our argument here applies to each agent individually, but that clearly implies our condition which is only predicated for groups, by repeated application of individual changes and the fact that the mechanism is non-bossy and individual preferences on consequences are strict.

If money transfers are allowed (Miyagawa, 2001), a variant of the above is again a group strategy-proof mechanism, generating the corresponding social choice function. But now individuals express their preferences over the pairs of houses and the (eventually personalized) prices they must pay for them. These preferences must be monotonic in money, and may now present indifferences. The relevant social choice function is derived from a natural adaptation of the top trading cycle, that includes a fixed order of objects to be used as tie-breaking rule among indifferent alternatives and provides well defined assignments for each preference profile. Again, an extension of the preferences on pairs of houses and prices to full allocations provides the domain of preferences over alternatives.

If money transfers are allowed, since no restrictions are imposed on the agents' preferences over houses and preferences are monotonic with respect to money, the domain of definition satisfies our richness domain condition.

To check for respectfulness, we shall use the following fact. Let $R_{i}$ and $R_{i}^{\prime}$ be a reshuffling of each other around the indifference class of a given consequence. Let $P_{i}$ and $P_{i}^{\prime}$ be the strict preferences resulting from using the same tie-breaking rule at $R_{i}$ and $R_{i}^{\prime}$. Then, $P_{i}$ and $P_{i}^{\prime}$ are reshuffling of each other around the same consequence. By definition of the top trading cycle, the consequences for the agent changing her preferences remain unchanged. By nonbossiness of the top trading cycle applied to the corresponding strict preference profile, all agents obtain the same consequence, and respectfulness follows. The same holds for joint monotonicity since an improvement of a given consequence in the preferences including indifferences is again either an improvement or a reshuffling of the same consequence for the corresponding strict preferences. In any case, and by definition of the top trading cycle and non-bossiness we can use an argument similar to the one we made for the case without monetary transfers.

One good auctions (Vickrey, 1961) There is one seller and a given number of buyers. The seller wants to give away one good in exchange for a monetary transfer. An auction is given by a set of rules that determines what actions must the seller and the eventual 
buyers use in order to arrive at a transaction, and what positive or negative transfers will be made to each partner involved. ${ }^{19}$ Agents are the seller and the buyers. Alternatives are full specifications of who gets the good, and what net transfers are made. Consequences for each buyer are whether or not they get the good, and the net transfer she obtains. Obtaining the good is typically assumed to be better than not, at the same level of transfers, and preferences are assumed to be separable and monotonic in money. Extending the preferences of selfish agents from consequences to alternatives provides us with the domain of preferences for the social choice function that assigns the result of an auction to each preference profile. For simplicity, we assume that the seller's reservation price is zero and that all buyers value the good positively.

Buyers are ranked according to their willingness to pay, and ties among those who want to pay the same amount are broken by a given hierarchy of buyers.

We describe the social choice function generated by Vickrey's second price auction as follows. Agents are ranked by their willingness to pay, and if some buyers have the same they are ranked according to a given hierarchy. The highest ranked agent gets the object, and pays what the second ranked buyer was willing to pay for it. The resulting social choice function is $H$-weakly group strategy-proof for the set $H$ of buyers, and satisfies our three conditions.

Now, we claim that in the standard Vickrey (1961) auction framework the domain of preferences for buyers is rich. One should only note that for any given pair of preference relations, the required intermediate preference in the definition of richness coincides with one of two we start with.

In order to check for buyers' respectfulness and joint monotonicity, we distinguish between the cases, depending on whether the buyer that changes her preference relation gets the object or not.

To check for respectfulness, first notice that if a buyer is not getting the object, there is no other reshuffling of her preferences around the original consequence than this preference itself. Next, assume that a buyer gets the object and pays for a second bid that is strictly lower than hers: then, no reshuffling can affect the consequences that she gets. The remaining case is the one where several buyers have the same highest bid and the tie-breaking hierarchy applies. But then, again, there is no reshuffling of the preferences of the buyer who gets the object around the original consequence other than her initial preference relation.

\footnotetext{
${ }^{19}$ Here again, as in the preceding examples, we stick to a simple formulation, for illustrative purposes.
} 
To check for buyers' joint monotonicity, take any subset of buyers and suppose that the consequences they get at a initial profile improve in their respective rankings. For a buyer who did not get the object in the first place, the fact that her consequence (no object, no payment) gets higher in her ranking means that her willingness to pay for the object has decreased. For a buyer who was getting the object in the first place, having her initial consequence (object, payment) become higher ranked means that her willingness to pay increases. The identity of the buyer getting the object in the Vickrey auction will not change in any of these cases: that particular buyer will end up paying less and therefore being strictly better off, while the rest of buyers involved in the change will get the same consequence as before and remain indifferent.

\section{Our contribution in perspective}

The purpose of this section is to provide some more perspective on our work, connecting it with different important contributions by other authors, explaining what we have done and why, and pointing at some additional research of ours that addresses related questions.

As we have already pointed out in Section 2, the incentives for groups of agents to cooperate in manipulating a mechanism can be examined with the help of a variety of tools and definitions. We have presented those of weak and strong strategy-proofness in Section 2 , but already warned the reader that other variants of this same concept can be defined. In addition, similar but related ideas have been developed by other authors, or could be studied in further work. Let us comment on those that we find closer to the spirit of the present paper.

Once we allow for several agents to participate in a joint manipulative action, the question arises whether or not all members of the group must actively misrepresent their preferences. The answer to this question is not substantial to establish whether a function is weakly group strategy-proof. This is because if a coalition can manipulate to the strict benefit of all of its members, including some that do not change their declaration, then the subsets of those agents that in fact misrepresent theirs can still strongly manipulate. However, when considering weak manipulations, that improve some of the members of a group while leaving others at the same level of satisfaction, it makes a difference whether or not one requires participants to actively misrepresent their preferences. Each specification in that respect leads to a different notion of strategy-proofness. The one allowing for more manipulations is the one where nothing is asked from the participating agents' actions, 
and corresponds to our definition of strong strategy-proofness. An intermediate version would require from participants in a group manipulation to actively misrepresent, whether they are to gain from it or not. We are not insisting in that intermediate notion of group strategy-proofness, but just mention it here to comment on some of the subtleties involved when formalizing the general idea.

Other possible ways to define the consequences of manipulative action by groups involve further departures from the idea of joint dominant strategies. If one considers that a potential manipulation is a threat, then some threats may be more serious than others. For example, one could think of counterthreats, and only take into account those threats that have no convincing counterthreats. We shall not discuss in detail the variety of refinements of the notions of group and individual strategy-proofness that would result from pursuing this line. Let us just mention that the underlying ideas are similar to those that lead to different notions of the bargaining set, and that they can be traced to very early treatments of the issue of manipulation (Farquharson, 1969, Pattanaik, 1976a, 1976b, and Barberà, 1980). More specifically, one could consider that, in the absence of enforcement mechanisms, agreements for jointly deviating that could further improve the lot of those who do not comply with them, if some members of the group do, should not be considered credible. This, again, suggests an attractive line of study. ${ }^{20}$ All of these departures point at weakenings of the notion of group strategy-proofness. Other versions of robustness of our generic notion would work in the opposite directions. Different authors have pointed out that even strategy-proof mechanisms can be effectively manipulated if monetary bribes are possible (Crémer, 1996, Schummer, 2000). The point has been made especially in connection with mechanisms that are weakly strategy-proof, like the second price auction: there, the second price bidder can improve the lot of the first price bidder by submitting a lower offer. Since the player whose preference change is crucial does not gain from it, the manipulation is weak. However, this paves the way for the high bidder to bribe the second one. Similar considerations can be extended further, and avoiding manipulations that could be induced through bribes would require even more than strong group strategy-proofness. The literature on the subject, which is of great practical importance, builds on extended models and mechanisms beyond the ones we have considered here. For early treatments of these issues see Graham and Marshall (1987), Roth and Sotomayor (1990), and Mailath and Zemsky (1991). What we had to say is about models where bribes have not yet entered the picture, and we think that these further considerations should not be superimposed on

\footnotetext{
${ }^{20}$ We are grateful to an anonymous referee for this comment.
} 
the models we deal with.

Let us also briefly comment on the connections between our work and the literature on implementation. We have not insisted on the notion of implementation, nor in the different solution concepts that can be used to make it specific, but concentrated in the use of dominant strategies by individuals, and the further idea of group strategy-proofness. Moreover, we have restricted attention to social choice functions rather than social choice correspondences, which are the objects on which implementation theory has obtained more positive results. Even then there are instances of well defined social choice functions that arise as the result of sophisticated play on the part of agents, for which our conditions may hold and our theorem may have consequences. Examples of such social choice functions are those induced by successive veto (Moulin, 1994, Moulin and Shenker, 1992) or by the use of different tree structures to arrive at majoritarian decisions (Miller, 1977, Barberà and Gerber, 2014). When applied to such functions, our theorem may lead to further conclusions about the incentives they provide, that we leave for further investigation.

It is also relevant to comment on the connection between the results of the present paper and those in our preceding one regarding the relationship between individual and weak group strategy-proofness. Here we insist in examples that involve private components in the consequences of agents, and were thus not covered by our previous paper for the case of pure public goods (Barberà, Berga, and Moreno, 2010). There we established that the equivalence between the two properties is precipitated by a single condition on the domain of the social choice functions. Here, we combine a domain condition with two others, that time on the social choice functions. The model, conditions and results in the present paper apply in particular to the public goods case. Hence, the results in our two papers are different from each other, and complementary, because of the different requirements that we use to guarantee equivalence in each one of them.

Notice also that the idea of combining strategy-proofness with other properties has been exploited by different authors, in search of additional interesting consequences. Fleurbaey and Maniquet (1997) proved that under an appropriate domain requirement, fair allocations where agents do not envy others will be attained by functions satisfying equal treatment of equals, non-bossiness and strategy-proofness, thus adding an interesting normative justification to the standard reasons to demand the latter condition. Another paper that connects strategy-proofness and fairness considerations in a similar spirit is Moulin (1993) who proves that within an appropriate domain strong group strategy-proof and anonymous social choice functions satisfy no envy. 
Even closer to our present concern is the following result that was first stated in Dasgupta, Hammond, and Maskin (1979) and later on reproduced in Maskin and Sjöström (2002, Theorem 7). A Maskin monotonic social choice function $f$ on a rich domain is weakly group strategy-proof. Their notion of richness is not the same of ours. But the main difference with our result is that Maskin monotonicity is assumed in the preceding statement, while we do not require this condition. This makes a big difference, because that condition implies, along with the richness of the domain, that the corresponding social choice function must be non-bossy. And that immediately excludes several of the mechanisms we have covered in our theorem, and in particular the Gale-Shapley social choice function.

We now want to return to one of the main issues addressed in the paper: the question whether there is a necessary conflict between incentives and efficiency. But since part of that discussion involves the interplay between different properties of social choice functions, we start with some comments on the weakness and the attractiveness of the properties we use in our main result.

Notice that different properties that are considered natural in the literature are stronger than the ones we have considered here. Weakening these properties has allowed us to encompass a large class of mechanisms, some of which would have been excluded ex-officio had we insisted in stronger requirements.

There is no doubt that some richness assumption is needed for any relevant analysis of allocation mechanisms, since we are interested in their performance under a variety of circumstances. Different authors, in different contexts, have defined richness in ways that suit the purposes of their analysis (see, for example, the notions of richness in Chatterji and Sen, 2011 or in Dasgupta, Hammond, and Maskin, 1979, or in the literature on VCG mechanisms), all of them different from ours and from each other. We have chosen our notion of richness rather empirically, as a common denominator for the variety of different environments and mechanisms that we wanted to put together. Let us also emphasize that its formulation is chosen in such a way that one does not need to refer to an agent's best alternative (like in Chatterji and Sen, 2011): this allows us to include environments where a maximal element need not be defined due to the presence of money.

Monotonicity is, in general terms, an attractive property. We have already pointed out that there exist several versions of it. Here we shall argue that our formulation of it is weaker than Maskin's, that we now define.

Definition 10 A social choice function $f$ satisfies Maskin monotonicity on $\times_{i \in N} \mathcal{R}_{i}$ if 
for any $R_{N} \in \times_{i \in N} \mathcal{R}_{i}$, any $i \in N$, and $R_{i}^{\prime} \in \mathcal{R}_{i}$ such that $\bar{U}\left(R_{i}, f_{i}\left(R_{N}\right)\right) \supseteq \bar{U}\left(R_{i}^{\prime}, f_{i}\left(R_{N}\right)\right)$, then $f_{j}\left(R_{i}^{\prime}, R_{-i}\right)=f_{j}\left(R_{N}\right)$ for each $j \in N$.

This version of Maskin's condition involves only the change of preferences by one agent at a time, while our joint monotonicity condition starts by assuming that several agents have changed from one profile to another. This is inconsequential, because Maskin's condition can be applied repeatedly and its consequences can be predicated for a group change as well. To establish a comparison with our monotonicity notion, consider the case where $a$ is the initially chosen alternative, and now its indifference class and that of their immediate followers in the preference order do merge. In that case, Maskin monotonicity still applies, but ours does not. Notice that this case (which is definitely well defined when the set of consequences is finite) is one where in fact the position of $a$ has definitely not improved. By excluding this case from consideration, our condition becomes not only weaker, but also more attractive normatively. A second difference is also consequential and represents another weakening away from Maskin monotonicity. The latter requires that, after appropriate preference changes by the members of a group, they all receive the same consequences, and all other agents must also stay with the same that they got initially. But our condition does not have this strong implication: it imposes no restriction on the consequences for agents whose preferences do not change, and allows those who change to eventually improve.

Because of that, we feel that our condition becomes quite palatable, even if, as we already said when defining it, there are weaker requirements in the same spirit. As for the fact that we compare profiles where the preferences of several agents differ (hence the qualification of joint monotonicity), this is due to the fact that there are strategy-proof mechanisms under which the repeated application of a similar condition for single agents would not lead to respect neither the joint requirement nor weak group strategy-proofness. Example 3 in the Appendix proves that this may be the case.

We also consider respectfulness to be normatively attractive, since it prohibits agents' changes in preferences to have a utility impact on the consequences for others unless they also have a utility impact on themselves. This notion is satisfied by the Gale-Shapley social choice function, and also by the one that derives from the assignment of one good through the Vickrey auction. Yet, both of these mechanisms would fail the stronger test of being non-bossy. For the case of Gale-Shapley see Example 7 in the Appendix (see also Kojima, 2010). In the case of Vickrey, this is because the second bidder could improve the lot of the high bidder by just bidding less, at no cost. 
Let us now return to the fundamental question of efficiency. The failure of ClarkeGroves mechanisms to avoid the waste of resources provides a leading example of situations where good incentives and efficiency are in conflict. Fortunately, this conflict is not always so acute. All mechanisms satisfying group strategy-proofness of some sort, for given environments, are instances where that classical dilemma becomes less dramatic or even non-existing. Specifically, it disappears in all environments where satisfactory mechanisms that are strong group strategy-proof can be identified. We have provided several examples of them, like division and house allocation without money. In other cases, strong group strategy-proofness is not achieved by the most well known mechanisms and some potential inefficiencies remain possible, but the allocations we get are at least weakly efficient (like matching, house allocation with money, cost sharing of a public good, and one good auctions). Our main theorem refers to the connection between individual and weak group strategy-proofness because we wanted to insist on the common features of all mechanisms satisfying at least that weakest demand on group incentives and we were aware that asking for more would again eliminate some important solutions to important problems.

Finally, a similar result to the one we report here could be envisaged: what properties of the domains and the functions could precipitate a direct equivalence between individual and strong group strategy-proofness? We have found a set of such properties: obviously they are not satisfied by some of the rules discussed here, but they are satisfied by others, like those we have considered as solutions to division and house allocation problems without money. The interested reader will find them in Barberà, Berga, and Moreno (2015).

Our main message is a plea for systematic consideration of group strategy-proofness as a very attractive property, that avoids the threat of manipulations by small groups, and at the same time reduces the potential conflict between incentives and efficiency. It is a positive message, because it proves that this coincidence is not only possible, but also based in the fact that many environments and mechanisms share a common set that conditions that allow for this attractive property to be met.

\section{Ackowledgements}

S. Barberà acknowledges support from the Severo Ochoa Programme for Centers of Excellence in R\&D (SEV-2011-0075), and grants "Consolidated Group-C" ECO2008-04756 and FEDER, ECO2014-53052-P, and SGR2014-515. D. Berga acknowledges the support from grants ECO2010-16353, ECO2013-45395-R, SGR2009-0189 and 2014-SGR-1360. B. Moreno acknowledges financial support from grants SEJ4941, SEJ-5980, ECO2011-29355 
and ECO2014-53767. We are specially grateful to the referees. The authors declare that they have np relevant or materiaal financial interests that relate to the research described in this paper.

\section{References}

[1] S. Barberà. "Stable Voting Schemes". Journal of Economic Theory 23 (2): 267-274 (1980).

[2] S. Barberà, D. Berga, and B. Moreno. "Individual versus group strategy-proofness: when do they coincide?". Journal of Economic Theory 145: 1648-1674 (2010).

[3] S. Barberà, D. Berga, and B. Moreno. "Group strategy-proofness in private good economies without money: matching, division and house allocation". Barcelona GSE Working Paper Series, Working Paper no 773 (2014).

[4] S. Barberà, D. Berga, and B. Moreno. "Sufficient conditions to reconcile strategyproofness with full efficiency". Mimeo September 2015.

[5] S. Barberà, and A. Gerber. "Sequential Voting and Agenda Manipulation". Barcelona GSE Working Paper No. 782 (2014).

[6] S. Barberà, M. Jackson, and A. Neme. "Strategy-Proof Allotment Rules". Games and Economic Behavior 18: 1-21 (1997).

[7] S. Barberà, H. Sonneschein, and L. Zhou. "Voting by Committees". Econometrica 59 (3): 595-609 (1991).

[8] C. Bird. "Group incentive compatibility in a market with indivisible goods." Economics Letters 14: 309-313 (1984).

[9] K. C. Border, and J. S. Jordan. "Straightforward elections, unanimity and phantom voters". Review of Economics Studies 50 (1): 153-170 (1983).

[10] S. Chatterji and A. Sen, "Tops-Only Domains", Economic Theory 46: 255-282 (2011).

[11] J. Crémer. "Manipulations by Coalitions Under Asymmetric Information: The Case of Groves Mechanisms". Games and Economic Behavior 13: 39-73 (1996). 
[12] P. Dasgupta, P. Hammond, and E. Maskin. "The Implementation of Social Choice Rules: Some General Results on Incentive Compatibility". The Review of Economic Studies 46 (2): 185-216 (1979).

[13] L. E. Dubins, and D. A. Freedman. "Machiavelli and the Gale-Shapley Algorithm". The American Mathematical Monthly 88 (7): 485-494 (1981).

[14] H. I. Ergin. "Efficient resource allocation on the basis of priorities". Econometrica 70 (6): 2489-2497 (2002).

[15] R. Farquharson. Theory of Voting, New Haven (1969).

[16] M. Fleurbaey and F. Maniquet. "Implementability and Horizontal Equity Imply NoEnvy". Econometrica 65: 1215-1219 (1997).

[17] D. Gale, and L. Shapley. "College Admissions and the Stability of Marriage". American Mathematical Monthly 69: 9-15 (1962).

[18] D. A. Graham, and R. C. Marshall. "Collusive Bidder Behavior at Single-Object Second-Price and English Auctions". The Journal of Political Economy 95 (6): 12171239 (1987).

[19] J. W. Hatfield, and F. Kojima. "Group Incentive Compatibility for Matching with Contracts". Games and Economic Behavior 67: 745-749 (2009).

[20] J. W. Hatfield, and P. R. Milgrom. "Matching with Contracts". American Economic Review 95 (4): 913-935 (2005).

[21] A. S. Kelso, Jr and V. P. Crawford. "Job Matching, Coalition Formation and Gross Substitutes". Econometrica 50 (6): 1483-1504 (1982).

[22] F. Kojima. "Impossibility of stable and nonbossy matching mechanisms". Economics Letters 107 (1): 69-70 (2010).

[23] G. J. Mailath and P. Zemsky. "Collusion in Second Price Auctions with Heterogeneous Bidders". Games and Economic Behavior 3: 467-486 (1991).

[24] R. Martínez, J. Massó, A. Neme, and J. Oviedo. "On group strategy-proof mechanisms for a many-to-one matching model". International Journal of Game Theory 33: 115128 (2004). 
[25] E. Maskin, and T. Sjöström. "Implementation Theory". In Handbook of Social Choice and Welfare, Volume 1, Edited by K.J Arrow, A.K. Sen and K. Suzumura, Elsevier Science (2002).

[26] N. R. Miller. "Graph-Theoretical Approaches to the Theory of Voting". American Journal of Political Science 21: 769-803 (1977).

[27] E. Miyagawa. "House allocation with transfers". Journal of Economic Theory 100: 329-355 (2001).

[28] H. Moulin. "On strategy-proofness and single peakedness". Public Choice 35: 437-455 (1980).

[29] H. Moulin. "On the Fair and Coalition-strategyproof Allocation of Private Goods". In Frontiers in Game Theory, Edited by K. Binmore, A. Kirman and P. Tani, Cambridge, Mass.: MIT Press (1993).

[30] H. Moulin. "Serial Cost Sharing of excludable public goods". Review of Economic Studies 61: 305-325 (1994).

[31] H. Moulin, and S. Shenker. "Serial Cost Sharing". Econometrica 60 (5): 1009-1037 (1992).

[32] S. Pápai. "Strategyproof Assignment by Hierarchical Exchange". Econometrica 68 (6): 1403-1433 (2000).

[33] P. K. Pattanaik. "Counter-threats and Strategic Manipulation under Voting Schemes". The Review of Economic Studies 43 (1): 11-18 (1976).

[34] P. K. Pattanaik. "Threats, Counter-Threats, and Strategic Voting". Econometrica 44 (1): 91-103 (1976).

[35] E. M. Penn, J. W. Patty, and S. Gailmard. "Manipulation and Single-Peakedness: A General Result". American Journal of Political Science 55 (2): 436-449 (2011).

[36] Z. Ritz. "Restricted Domains, Arrow Social Welfare Functions and Noncorruptible and Nonmanipulable Social Choice Correspondences: The Case of Private and Public Alternatives". Journal of Economic Theory 35: 1-18 (1985). 
[37] A. Roth. "The Economics of Matching: Stability and Incentives". Mathematics of Operations Research 7 (4): 617-628 (1982).

[38] A. Roth, and M. Sotomayor. Two-sided Matching: A Study in Game-Theoretic Modeling and Analysis. Cambridge University Press, Cambridge, England. [Econometrica Society Monographs No. 18] (1990).

[39] M. A. Satterthwaite, and H. Sonnenschein. "Strategy-Proof Allocation Mechanisms at Diferentiable Points". Review of Economic Studies 48: 587-597 (1981).

[40] J. Schummer. "Manipulation through bribes". Journal of Economic Theory 91: 180198 (2000).

[41] S. Serizawa. "Strategy-Proof and Symmetric Social Choice Functions for Public Good Economies". Econometrica 67 (1): 121-145 (1999).

[42] L. Shapley, and H. Scarf. "On Cores and Indivisibilities". Journal of Mathematical Economics 1: 23-37 (1974).

[43] Y. Sprumont. "The division problem with single-peaked preferences: A characterization of the uniform allocation rule". Econometrica 59: 509-519 (1991).

[44] W. Thomson. "Non-bossiness". Mimeo. University of Rochester (2014).

[45] R.A. Velez. "Consistent strategy-proof assignment by hierarchical exchange." Economic Theory 56: 125-156 (2014).

[46] W. Vickrey. "Counterspeculation, Auctions, and Competitive Sealed Tenders". Journal of Finance 16: 8-37 (1961).

\section{Appendix: Examples}

Example 1 Violation of Theorem 1 when the social choice function is $H$-joint monotonic, $H$-strategy-proof, $H$-respectful but not $H$-weak group strategy-proof and the domain is not $H$-rich. Consider a one-to-one matching model. Let $W=\{1,2,3\}$ and $F=\{4,5,6\}$. For each $j \in F, B_{j}=W \cup\{j\}$ and for agent $3 \in W, B_{3}=F \cup\{3\}$ any linear order on $B_{l}$ is 
admissible. For agents 1 and $2 \in W, B_{i}=F \cup\{i\}$, the set of admissible preferences are as follows:

\begin{tabular}{|l|l|l||l|l|l|}
\hline$R_{1}^{1}$ & $R_{1}^{2}$ & $R_{1}^{3}$ & $R_{2}^{1}$ & $R_{2}^{2}$ & $R_{2}^{3}$ \\
\hline 4 & 5 & 6 & 4 & 5 & 6 \\
\hline 5 & 4 & 4 & 5 & 4 & 5 \\
\hline 6 & 6 & 5 & 6 & 6 & 4 \\
\hline 1 & 1 & 1 & 2 & 2 & 2 \\
\hline
\end{tabular}

Define the following rule $f$ where neither firms nor agent 3 play any role, that is:

\begin{tabular}{|l|l|l|l|}
\hline$f\left(\cdot, R_{3}, R_{F}\right)$ & $R_{2}^{1}$ & $R_{2}^{2}$ & $R_{2}^{3}$ \\
\hline$R_{1}^{1}$ & $4,5,6,1,2,3$ & $4,5,6,1,2,3$ & $5,6,4,3,1,2$ \\
\hline$R_{1}^{2}$ & $5,4,6,2,1,3$ & $5,4,6,2,1,3$ & $5,6,4,3,1,2$ \\
\hline$R_{1}^{3}$ & $6,4,5,2,3,1$ & $6,4,5,2,3,1$ & $5,4,6,2,1,3$ \\
\hline
\end{tabular}

For $H=W$, the domain is not $H$-rich (let $R_{1}=R_{1}^{3}, \widetilde{R}_{1}=R_{1}^{2}, b_{i}=4$ and $a_{i}=5$, there does not exist any $R_{1}^{\prime}$ satisfying the required condition). And the above rule is $H$-respectful, $H$-joint monotonic, $H$-strategy-proof, but it is not $H$-weak group strategy-proof (agents 1 and 2 deviates from $\left(R_{1}^{3}, R_{2}^{3}\right)$ to $\left(R_{1}^{1}, R_{2}^{1}\right)$ and they are strictly better off).

Example 2 Violation of Theorem 1 when the social choice function defined on a H-rich domain is $H$-joint monotonic, $H$-strategy-proof but neither $H$-respectful nor $H$-weak group strategy-proof. Consider a one-to-one matching model. Let $H=W, W=\{1,2\}$ and $F=\{3,4\}$. For each $j \in F, B_{j}=W \cup\{j\}$ and any linear order on $B_{j}$ is admissible. For each $i \in W, B_{i}=F \cup\{i\}$ and any linear order on $B_{i}$ is admissible. The latter is as follows:

\begin{tabular}{|l|l|l|l|l|l|}
\hline$R_{i}^{1}$ & $R_{i}^{2}$ & $R_{i}^{3}$ & $R_{i}^{4}$ & $R_{i}^{5}$ & $R_{i}^{6}$ \\
\hline 3 & 4 & 3 & 4 & $i$ & $i$ \\
\hline 4 & 3 & $i$ & $i$ & 3 & 4 \\
\hline$i$ & $i$ & 4 & 3 & 4 & 3 \\
\hline
\end{tabular}


Define the following rule $f$ where firms do not play any role, that is for any $R_{F} \in \mathcal{R}_{F}$ :

\begin{tabular}{|l|l|l|l|l|l|l|}
\hline$f\left(\cdot, R_{F}\right)$ & $R_{2}^{1}$ & $R_{2}^{2}$ & $R_{2}^{3}$ & $R_{2}^{4}$ & $R_{2}^{5}$ & $R_{2}^{6}$ \\
\hline$R_{1}^{1}$ & $3,4,1,2$ & $3,4,1,2$ & $3,2,1,4$ & $3,4,1,2$ & $4,2,3,1$ & $4,2,3,1$ \\
\hline$R_{1}^{2}$ & $4,3,2,1$ & $4,3,2,1$ & $4,3,2,1$ & $4,2,3,1$ & $4,2,3,1$ & $4,2,3,1$ \\
\hline$R_{1}^{3}$ & $3,4,1,2$ & $3,4,1,2$ & $3,2,1,4$ & $3,4,1,2$ & $4,2,3,1$ & $4,2,3,1$ \\
\hline$R_{1}^{4}$ & $4,3,2,1$ & $4,3,2,1$ & $4,3,2,1$ & $4,2,3,1$ & $4,2,3,1$ & $4,2,3,1$ \\
\hline$R_{1}^{5}$ & $1,3,2,4$ & $1,3,2,4$ & $1,3,2,4$ & $1,3,2,4$ & $4,3,2,1$ & $4,3,2,1$ \\
\hline$R_{1}^{6}$ & $1,3,2,4$ & $1,3,2,4$ & $1,3,2,4$ & $1,3,2,4$ & $4,3,2,1$ & $4,3,2,1$ \\
\hline
\end{tabular}

For any $R_{F} \in \mathcal{R}_{F}$, note that $f\left(R_{1}^{5}, R_{2}^{5}, R_{F}\right)=(4,3,2,1)$. Observe that $f_{1}\left(R_{1}^{5}, R_{2}^{5}, R_{F}\right)=$ $f_{1}\left(R_{1}^{3}, R_{2}^{5}, R_{F}\right)=4, \bar{U}\left(R_{1}^{5}, 4\right)=\bar{U}\left(R_{1}^{3}, 4\right), \bar{L}\left(R_{1}^{5}, 4\right)=\bar{L}\left(R_{1}^{3}, 4\right)$ but $f_{2}\left(R_{1}^{5}, R_{2}^{5}, R_{F}\right) \neq f_{2}\left(R_{1}^{3}, R_{2}^{5}, R_{F}\right)$, violating $H$-respectfulness. Observe that $f$ is strongly manipulable at $\left(R_{1}^{5}, R_{2}^{5}, R_{F}\right)$ by coalition $C=\{1,2\} \subseteq H$ via $\left(R_{1}^{1}, R_{2}^{3}\right)$, thus violating $H$-weak group strategy-proofness.

Example 3 Violation of Theorem 1 when the social choice function defined on a rich domain is strategy-proof, respectful, but neither joint monotonic nor weak group strategyproof. Let $N=\{1,2\}$, agents's consequences $B_{1}=B_{2}=\{a, b, c, d\}$, and the set of admissible preferences of each agent over consequences be the same: $\mathcal{R}_{1}=\mathcal{R}_{2}=\left\{R, R^{\prime}\right\}$, where $R$ : cIdPaIb and $R^{\prime}: a I^{\prime} b P^{\prime} c I^{\prime} d$. Observe that $\mathcal{R}_{1} \times \mathcal{R}_{2}$ is an $N$-rich domain. Let $f$ be the social choice function defined as follows: $f\left(R_{1}, R_{2}\right)=(b, b), f\left(R_{1}, R_{2}^{\prime}\right)=(c, a)$, $f\left(R_{1}^{\prime}, R_{2}\right)=(a, c)$, and $f\left(R_{1}^{\prime}, R_{2}^{\prime}\right)=(d, d)$. Observe that $f$ is strategy-proof and respectful (in a vacuous way). ${ }^{21}$ However, $f$ violates weak group strategy-proofness: $f\left(R_{1}^{\prime}, R_{2}^{\prime}\right)=(d, d)$ but both agents would be strictly better off obtaining $b$ which would be their assignment if their preferences were $\left(R_{1}, R_{2}\right)$. Furthermore, $f$ violates joint monotonicity: when going from profile $\left(R_{1}^{\prime}, R_{2}^{\prime}\right)$ to profile $\left(R_{1}, R_{2}\right)$ we have that for each $i \in N, \bar{L}\left(R_{i}^{\prime}, d\right) \subseteq \bar{L}\left(R_{i}, d\right)$ and $\bar{U}\left(R_{i}, d\right) \subseteq \bar{U}\left(R_{i}^{\prime}, d\right)$, but $f_{i}\left(R_{1}^{\prime}, R_{2}^{\prime}\right)=d P_{i} b=f_{i}\left(R_{1}, R_{2}\right)$ which contradicts joint monotonicity. Note though that $f$ would satisfy a weaker version of joint monotonicity requiring only individual preference changes (see a discussion about it in Section 5).

Example 4 A social choice function defined on a $H$-rich domain (of strict preferences over consequences) that is $H$-respectful, $H$-joint monotonic but not $H$-strategy-proof. Consider a one-to-one matching model. Let $H=W$. The much celebrated Boston mechanism provides an example. In a first round, each student applies to her (reported) top choice and each

\footnotetext{
${ }^{21}$ Note that $f$ also satisfies non-bossiness.
} 
school admits applicants one at a time according to its preferences until either capacity is exhausted or there are no more students who ranked it first. In Round $k$, each unmatched student applies to her $k$ th choice and schools with remaining capacity admits applicants one at a time according to its preferences until either the remaining capacity is exhausted or there are no more students who ranked it $k$ th.

Example $5 A$ social choice function defined on a $H$-rich domain that is $H$-respectful, $H$ strategy-proof but not $H$-joint monotonic. Consider a one-to-one matching model. Let $H=W, W=\{1,2,3\}$ and $F=\{4,5,6\}$. For each $i \in W, B_{i}=F \cup\{i\}$ and any linear order on $B_{i}$ is admissible. For each $j \in F, B_{j}=W \cup\{j\}$ and any linear order on $B_{j}$ is admissible. For any $R_{1} \in \mathcal{R}_{1}$, let $\tau^{2}\left(R_{1}\right)$ be the most preferred alternative on $B_{1} \backslash \tau\left(R_{1}\right)$ of $R_{1}$. Define the following rule $f$ where neither firms nor agents in $W \backslash\{1\}$ play any role:

\begin{tabular}{|l|l|}
\hline & $\forall R_{-1}, f\left(R_{N}\right)$ \\
\hline If $\tau\left(R_{1}\right)=5$ then & $5,6,4,3,1,2$ \\
\hline If $\tau\left(R_{1}\right)=6$ then & $6,5,4,3,2,1$ \\
\hline If $\tau\left(R_{1}\right)=1$ then & $1,5,4,3,2,6$ \\
\hline If $\tau\left(R_{1}\right)=4$ and $\tau^{2}\left(R_{1}\right)=5$ then & $5,4,6,2,1,3$ \\
\hline If $\tau\left(R_{1}\right)=4$ and $\tau^{2}\left(R_{1}\right)=6$ then & $6,4,5,2,3,1$ \\
\hline If $\tau\left(R_{1}\right)=4$ and $\tau^{2}\left(R_{1}\right)=1$ then & $1,4,5,2,3,6$ \\
\hline
\end{tabular}

For $H=W$, the above rule is $H$-respectful, $H$-strategy-proof (it is also $H$-group strategyproof), but it is not $H$-joint monotonic. To see this consider the following preference profile:

\begin{tabular}{|l|l|l|}
\hline$\widehat{R}_{1}$ & $\widehat{R}_{2}$ & $\widehat{R}_{3}$ \\
\hline 4 & 5 & 4 \\
\hline 1 & 4 & 5 \\
\hline 5 & 6 & 6 \\
\hline 6 & 2 & 3 \\
\hline
\end{tabular}

For any $R_{F} \in \mathcal{R}_{F}$, note that $f\left(\widehat{R}_{1}, \widehat{R}_{2}, \widehat{R}_{3}, R_{F}\right)=(1,4,5,2,3,6)$. Suppose that the preferences of agents in $H=W$ change and the consequences they have obtained are now the 
most preferred ones

\begin{tabular}{|l|l|l|}
\hline$\widetilde{R}_{1}$ & $\widetilde{R}_{2}$ & $\widetilde{R}_{3}$ \\
\hline 1 & 4 & 5 \\
\hline 4 & 5 & 4 \\
\hline 5 & 6 & 6 \\
\hline 6 & 2 & 3 \\
\hline
\end{tabular}

For any $R_{F} \in \mathcal{R}_{F}$, note that $f\left(\widetilde{R}_{1}, \widetilde{R}_{2}, \widetilde{R}_{3}, R_{F}\right)=(1,5,4,3,2,6)$. Observe that $f_{1}\left(\widetilde{R}_{1}, \widetilde{R}_{2}, \widetilde{R}_{3}, R_{F}\right)=$ $f_{1}\left(\widehat{R}_{1}, \widehat{R}_{2}, \widehat{R}_{3}, R_{F}\right)=1$ but $f_{i}\left(\widetilde{R}_{1}, \widetilde{R}_{2}, \widetilde{R}_{3}, R_{F}\right) \neq f_{i}\left(\widehat{R}_{1}, \widehat{R}_{2}, \widehat{R}_{3}, R_{F}\right)$ for $i=2,3$, violating $H$ joint monotonicity.

Example 6 A social choice function defined on a $H$-rich domain that is $H$-weak group strategy-proof but not $H$-respectful. Consider a one-to-one matching model. Let $H=W$, $W=\{1,2,3\}$ and $F=\{4,5,6\}$. For each $i \in W, B_{i}=F \cup\{i\}$ and any linear order on $B_{i}$ is admissible. For each $j \in F, B_{j}=W \cup\{j\}$ and any linear order on $B_{j}$ is admissible. For any $R_{1} \in \mathcal{R}_{1}$, let $\tau^{2}\left(R_{1}\right)$ be the most preferred alternative on $B_{1} \backslash \tau\left(R_{1}\right)$ of $R_{1}$ and $\tau^{3}\left(R_{1}\right)$ be the worst alternative on $B_{1}$ of $R_{1}$. Define the following rule $f$ where worker 1 dictates as follows: $f_{1}\left(R_{N}\right)=\tau\left(R_{1}\right), f_{2}\left(R_{N}\right)=\tau^{2}\left(R_{1}\right)$, and $f_{3}\left(R_{N}\right)=\tau^{3}\left(R_{1}\right)$. This rule $f$ is $H$-group strategy-proof but $f$ is not $H$-respectful: Let $R_{1}$ and $R_{1}^{\prime}$ such that $4 P_{1} 5 P_{1} 6$ and $4 P_{1}^{\prime} 6 P_{1}^{\prime} 5$. By definition of $f$, for any $R_{N \backslash\{1\}}, f_{1}\left(R_{1}, R_{N \backslash\{1\}}\right)=f_{1}\left(R_{1}^{\prime}, R_{N \backslash\{1\}}\right)=4, f_{2}\left(R_{1}, R_{N \backslash\{1\}}\right)=5 \neq$ $f_{2}\left(R_{1}^{\prime}, R_{N \backslash\{1\}}\right)=6$ and $f_{3}\left(R_{1}, R_{N \backslash\{1\}}\right)=6 \neq f_{3}\left(R_{1}^{\prime}, R_{N \backslash\{1\}}\right)=5, \bar{L}\left(R_{1}, f_{1}\left(R_{1}, R_{N \backslash\{1\}}\right)\right)=$ $\bar{L}\left(R_{1}^{\prime}, f_{1}\left(R_{1}, R_{N \backslash\{1\}}\right)\right)$, and $\bar{U}\left(R_{1}, f_{1}\left(R_{1}, R_{N \backslash\{1\}}\right)\right)=\bar{U}\left(R_{1}^{\prime}, f_{1}\left(R_{1}, R_{N \backslash\{1\}}\right)\right)=\varnothing$.

Example 7 The Gale-Shapley social choice function is bossy. Consider a one-to-one matching model. Let $H=W, W=\{5,6,7,8\}, M=\{1,2,3,4\}$, and for each $i \in W$, $B_{i}=M \cup\{i\}$ and for each $j \in M, B_{j}=W \cup\{j\}$. Consider the following strict preference profile $\left(R_{W}, R_{M}\right)$ where a column represents the strict preference of an agent.

\begin{tabular}{|l|l|l|l||l|l|l|l|}
\hline$R_{5}$ & $R_{6}$ & $R_{7}$ & $R_{8}$ \\
\hline 3 & 4 & 2 & 2 & $R_{1}$ & $R_{2}$ & $R_{3}$ & $R_{4}$ \\
\hline 1 & 3 & 4 & 4 & 6 & 7 & 8 \\
\hline 2 & 2 & 3 & 1 & 7 & 8 & 5 & 7 \\
\hline 4 & 1 & 1 & 3 & 7 & 6 & 6 \\
\hline 5 & 6 & 7 & 8 & 5 & 8 & 5 \\
\hline 1 & 2 & 3 & 4 \\
\hline
\end{tabular}

Let $R_{5}^{\prime}$ be 5's preference deviation be such that $1 P_{5}^{\prime} 3 P_{5}^{\prime} 2 P_{5}^{\prime} 4 P_{5}^{\prime} 5$. Applying the women's proposal Gale-Shapley mechanism to $\left(R_{W}, R_{M}\right)$ and $\left(R_{5}^{\prime}, R_{-W}, R_{M}\right)$ we obtain the matchings: 


$$
\begin{aligned}
& f\left(R_{W}, R_{M}\right)=\quad\left(\begin{array}{llll}
5 & 6 & 7 & 8 \\
1 & 2 & 3 & 4
\end{array}\right), \text { while } \\
& f\left(R_{5}^{\prime}, R_{-W}, R_{M}\right)=\left(\begin{array}{cccc}
5 & 6 & 7 & 8 \\
1 & 3 & 4 & 2
\end{array}\right) .
\end{aligned}
$$

Hence, the Gale-Shapley social choice function is bossy. Note also that the Gale-Shapley is $W$-weakly manipulable at $\left(R_{W}, R_{M}\right)$ by coalition $C=\{5,6\}$ via $R_{5}^{\prime}$. 